\title{
Neuropathic pain in HIV patients receiving ART without stavudine in an Indonesia Referral Hospital
}

Fitri OCTAVIANA (MD, PhD) ${ }^{a, b}$, Ahmad Yanuar SAFRI (MD) ${ }^{a, b}$, Denise Dewanto SETIAWAN (MD)a, Riwanti ESTIASARI (MD, PhD) ${ }^{a, b}$, Darma IMRAN (MD) a,b, Teguh RANAKUSUMA (MD) ${ }^{a, b}$, Patricia PRICE

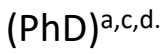

a Neurology Department, Faculty of Medicine, Universitas Indonesia, Jakarta, Indonesia

b Neurology Department, Cipto Mangunkusumo Hospital, Jakarta, Indonesia

c School of Biomedical Sciences, Curtin University, Bentley, Australia

d School of Physiology, University of the Witwatersrand, Johannesburg, South Africa

Short title: Neuropathic pain in HIV patients

Disclosure of Interest Statement: The project was funded by Curtin University and Universitas Indonesia. No pharmaceutical grants were received.

\section{Corresponding Author:}

A/Professor Patricia Price

School of Biomedical Science,

Curtin University,

Bentley, 6102

Australia

Tel: 618-92669716

Email: patricia.price@curtin.edu.au

Keywords: Pain, HIV, Sensory Neuropathy, stavudine

Word Count: Abstract 238 words, Text 1203 words 


\begin{abstract}
Lower limb neuropathic pain in HIV patients is a common manifestation of sensory neuropathy (HIVSN), but can be seen in patients who do not meet standard definitions of HIV-SN. The drug stavudine is a risk factor for HIV-SN, but some patients treated without stavudine experience HIV-SN, and the prevalence and risk factors influencing neuropathic pain in this setting are unknown. A cross sectional study at Cipto Mangunkusumo Hospital Jakarta tested 197 HIV patients treated for $>12$ months without stavudine. HIV-SN was defined using the AIDS Clinical Trial Group Brief Peripheral Neuropathy Screening Test (ACTG-BPNST). A validated Indonesia translation of Douleur Neuropathique en 4 (DN4) questionnaire was used to assess lower limb neuropathic pain. Nerve conduction studies assessed large nerve fiber function and Stimulated Skin Wrinkle (SSW) tests were performed to assess small nerve fibers. The prevalence of neuropathic pain was $6.6 \%$. BPNST ${ }^{+} H I V-S N$ was diagnosed in $14.2 \%$ of the cohort and $38.5 \%$ of patients with pain. Use of protease inhibitors and ART duration $<2$ years associated with neuropathic pain in univariate $(p=0.036, p=0.002$, resp.) and multivariable analyses (model $\mathrm{p}<0.001$ ). SSW tests were abnormal in $53.8 \%$ of subjects with neuropathic pain and only $25.5 \%$ without pain $(p=0.05)$. Patients with pain without BPNST ${ }^{+} H I V-S N$ had begun ART more recently than those with both diagnoses. Overall this preliminary study showed that neuropathic pain associated with protease inhibitors and a shorter duration of ART in Indonesian HIV patients, and may be an early symptom of small fiber neuropathy in this context.
\end{abstract}




\section{Introduction}

Neuropathic pain (NP) is a common symptom of sensory neuropathy in HIV patients (HIV-SN), occurring both in anti-retroviral therapy (ART) naïve patients(1) and among those using ART. NP can affect the adherence to ART and influence the quality of life (2), Nucleoside reverse transcriptase inhibitors (NRTI) such as stavudine (3), and Protease Inhibitors (PI) (4), have been associated with HIVSN, but their role in NP is less clear. In our studies assessing HIV-SN by AIDS Clinical Trial Group Brief Peripheral Neuropathy Screening Tool (ACTG-BPNST), the prevalence of HIV-SN halved between 2006 when all patients received stavudine (observed associations with HIV-SN were increasing age and height) and 2016 when this drug was no longer used. HIV-SN was then associated with ongoing HIV replication (5).

NP occurs in diverse clinical syndromes and has been linked with damage to small myelinated fibers or unmyelinated C-fibers [reviewed in (6)]. There is evidence that HIV may damage large myelinated fibers, with $\mathrm{CD}^{+}$T-cell counts correlating inversely with abnormal nerve conduction velocity in one study (7). In the same cohort, the duration of neurotoxic ART correlated with an abnormal Sympathetic Skin Response, reflecting damage to thin unmyelinated sympathetic nerve fibers and with reduced intraepithelial nerve fiber densities evident histologically (8).

We present a preliminary study assessing associations between NP and clinical factors, demographics, and small and large nerve fiber function in clinic patients with HIV receiving modern (stavudine sparing) ART regimens.

\section{Methods}

HIV-positive adults who had used ART for at least 12 months but who had never been exposed to stavudine were screened for neuropathy and neuropathic pain at the Integrated HIV Care Clinic, Cipto Mangunkusumo Hospital, Jakarta, Indonesia. Potential participants were screened by selecting from medical record of HIV patients who had attended the clinic for at least 1 year. Patients with any history of other conditions linked with a neuropathy (e.g. diabetes mellitus, Leprae, history of Guillain-Barré Syndrome, malignancy, radiculopathy) or unable to provide informed consent were 
excluded. Sensory neuropathy was assessed by a neurologist (FO) and a general practitioner (DDS) who had been trained by FO using the AIDS Clinical Trials Group Brief Peripheral Neuropathy Screen Tool (ACTG-BPNST), and defined as present if the individual had one or more of the lower limb neuropathic symptoms ( $\geq 1$ positive subjective symptom), plus absent ankle reflexes or reduced vibration sense at the great toe (positive objective sign). NP was defined as shooting, stabbing, sharp or burning pain of bilateral lower limbs and assessed using The Douleur Neuropathique en 4 (DN4) questionnaire that has been translated and validated in the Indonesian language (9). A numeric rating scale (NRS) was used to assess pain severity. Pain was classified as mild (NRS $\leq 5)$, moderate (NRS=6-7), or severe (NRS $\geq 8$ ). The study was approved by the Ethics Committee of the Faculty of Medicine, Universitas Indonesia (579/UN2.F1/ETIK/2014). All participants gave written informed consent.

Stimulated Skin Wrinkling (SSW) tests used eutectic mixture of local anesthetic cream contained 2.5\% lidocaine and $2.5 \%$ prilocaine to assess the function of small peripheral nerve fibers. SSW was performed on both hands. The cut-off point defining a normal response was 3 degrees of skin wrinkling. SSW was assessed as abnormal if both hands were affected (10). Nerve conduction studies (NCS) were performed by a neurologist (FO) and assessed motor and sensory nerves in the upper and lower limbs using a Sierra-Summit Cadwe $\|^{\circledR}$ instrument. Motor function was assessed bilaterally in the common peroneal and tibial nerves, right median and ulnar nerves. Antidromic sensory conduction was assessed in medial, ulnar, peroneal superficial and sural nerves. NCS was defined as abnormal if one or more of amplitude, distal latency or conduction velocity was abnormal bilaterally compared to normal values derived in our clinic.

Patient height and weight were measured during the study assessment and clinical data were collected from medical files. Plasma HIV RNA was measured using a Cobas Amplicor Monitor (Roche Molecular Diagnostics, Pleasanton, CA).

Statistical analyses utilized SPSS version 21.0 and GraphPad Prism 7.0. Demographic, clinical and treatment details of patients with and without neuropathy were compared using Fisher's exact tests (dichotomous variables), Mann-Whitney tests [non-parametric data, described using median (range)] or unpaired $t$ tests [parametric data, described using mean \pm SD] and Spearman's correlations (non- 
parametric continuous variables). Multivariable analyses were undertaken using logistic regression modeling (including all factors with $\mathrm{p}<0.25$ on univariate analysis) with a stepwise removal procedure.

\section{Results}

From 2596 HIV patients, 907 met inclusion criteria - including ART without stavudine for 12 months. However, of eligible patients, only 197 patients gave their consent. Patient selection is described in Supplementary Figure 1. These individuals were screened as described above. We identified 13 subjects (6.6\%) with NP and 28 (14.2\%) with HIV-SN assessed by BPNST (BPNST+HIV-SN). Eight among 13 subjects with NP had symptoms consistent with sensory neuropathy (positive subjective signs) with normal tendon reflexes and normal vibration tests (negative objective signs). The remaining 5 subjects were BPNST ${ }^{+} \mathrm{HIV}-\mathrm{SN}^{+}$.

Univariate associations with NP are presented in Table 1. The presence of NP associated with use of a protease inhibitor (PI; Lopinavir/Ritonavir), shorter ART duration (median 1.7 (1-6.4) years), the presence of BPNST $^{+}$HIV-SN or an abnormal SSW test $(p<0.05)$. Isoniazid use and alcohol consumption were not associated risk factors of NP. We observed a low rate of abnormal NCS in this cohort (6.5\%) and all patients with NP had normal NCS data. Multivariable analyses tested all factors that achieved $\mathrm{p}<0.25$ in univariate analysis, followed by a stepwise removal procedure. PI use [OR $(95 \% \mathrm{Cl}): 4.4(1.2-$ 16), $p=0.02$ ] and ART duration $<2$ years [OR $(95 \% \mathrm{Cl})$ : $6.9(2.1-23), p=0.002]$ were independently associated with NP and created a significant model $\left(R^{2}=0.18, p<0.001\right)$.

Pain was described as mild in 4 subjects and moderate-severe in 9 subjects. We noted negative correlations between pain score and nadir CD4 ${ }^{+} \mathrm{T}$-cell count $(r=-0.75, p=0.003)$ or current $C D 4^{+} \mathrm{T}$-cell count ( $r=-0.64, p=0.017)$. Accordingly, median nadir and current $C D 4^{+}$-cell counts were lower in patients with moderate-severe NP (54 vs. 309 cell/ $\mu \mathrm{L} ; \mathrm{p}=0.006$ and 242 vs. 527 cell/ $\mu \mathrm{L} ; \mathrm{p}=0.011$, respectively) than those with mild NP. Neither PI use nor ART duration associated with pain severity $(p=0.65)$.

As indication whether pain precedes BPNST ${ }^{+}$HIV-SN, we assessed time on ART. Patients with NP without BPNST ${ }^{+} \mathrm{HIV}-\mathrm{SN}$ had been on ART for a shorter period than those with NP and BPNST ${ }^{+} \mathrm{HIV}-\mathrm{SN}$ 
$(p=0.03)$ or BPNST $^{+}$HIV-SN only $(p=0.0009)$. The latter two groups had received ART for a similar time $(p=0.2)$ (Figure 1).

\section{Discussion}

The prevalence of NP is $20.9 \%$ among ART naïve HIV patients (11) and about $14.3 \%$ in HIV patients on ART with stavudine (12). Here the prevalence of NP was lower in patients treated without stavudine. Unfortunately, we have no equivalent data to assess whether NP is more common in our patient cohort than the general population of Jakarta. A meta-analysis (13) presents French, Brazilian and Canadian studies with a high prevalence of pain (7-18\%) assessed with DN4 but notes that all were postal or telephone surveys. Some or all may include traumatic nerve injury, arthritic pain and postherpetic pain, so the question of what is "normal" remains unresolved.

PI-based ART was independently associated with NP in our study and has been associated with HIV-SN previously (3). We saw only a marginal association between $\mathrm{CD}^{+} \mathrm{T}$-cell counts and prevalent NP $(p=0.09)$, but there was a clearer association between lower nadir and current CD4+ T-cell count and moderate-severe pain in patients with NP. Low $\mathrm{CD}^{+}{ }^{+}$T-cell counts are associated with systemic immune activation and associated inflammation around peripheral nerves. We recently demonstrated chemokine receptors expressed by $\mathrm{CD}_{1} 4^{+}$and $\mathrm{CD}^{+}$cells infiltrating tissues around damaged cutaneous nerves in HIV-SN patients (14).

Isoniazid is well known as a factor associated with peripheral neuropathy. Co-administration of isoniazid and stavudine increased incidence of HIV-SN when compared the use of stavudine alone (15) but in this cohort of patients with no exposure to stavudine, isoniazid was not associated with HIV-SN (5) or NP. High levels alcohol consumption is a risk factor of neuropathy (16) but was not associated with NP in this study. However, we didn't assess the quantity and type of alcohol consumed. Anecdotally, consumption is rarely high, but it may be under reported in the predominantly Muslim population of Jakarta.

Here 5/13 NP subjects were assessed as HIV-SN by BPNST. The DN4 questionnaire includes the assessment of small fiber nerves (pin-prick test) while the BPNST also assesses the large fiber nerve 
(deep tendon reflex, vibration test). Therefore, patients who only had small fiber nerve impairment might have negative result on BPNST. Accordingly, NCS were normal in all patients with NP in our study, consistent with small fibers being invisible to NCS. On the other hand, abnormalities in SSW did associate with NP in this cohort. SSW is a simple way to diagnose small fiber neuropathy in HIV patients with sensitivity of $60 \%$ and specificity of $67 \%$ compare to BPNST (17). Wrinkling of the fingertips results from vasoconstriction controlled by sympathetic fibers. It has been used to establish small fiber damage in diabetes and approaches the sensitivity of intraepidermal nerve fiber density (10).

Patients with NP who did not meet the criteria for a diagnosis of HIV-SN by BPNST had begun ART more recently than those with HIV-SN, so NP may be an early sign of HIV-SN, occurring before clinical signs assessed using the BPNST. Indeed NP can be an early symptom of diabetic neuropathy, which is pathologically similar to HIV-SN (18). However, the cross-sectional study design precludes assessment of causation and the small number of subjects with NP limited our power to assess associations.

The small number participants that were enrolled in this study clearly a limitation and findings warrant confirmation. Many patients were unwilling to enroll due to their long waiting times in the outpatient clinic and some refused the NCS due to their fear of electrical currents. However we accept that patients with symptoms may be more likely to enroll. However we show that HIV patients can experience NP without taking stavudine, but PI may be associated with this condition. We provide evidence that NP may be an early sign of HIV-SN, and recommend screening for NP and considering use of non-PI based ART in affected individuals.

\section{Acknowledgment}

Dr Catherine Cherry has contributed to this study with data analyses and clinical perspectives. 


\section{References}

1. Phillips TJC, Brown M, Ramirez JD, Perkins J, Woldeamanuel YW, Williams AC de C, et al. Sensory, psychological, and metabolic dysfunction in HIV-associated peripheral neuropathy: A crosssectional deep profiling study. Pain. 2014;155:1846-1860.

2. Keltner JR, Vaida F, Ellis RJ, Moeller-Bertram T, Fitzsimmons C, Duarte NA, et al. Health-Related Quality of Life 'Well-Being" in HIV distal neuropathic pain is more strongly associated with depression severity than with pain intensity.' Psychosomatics. 2012;53:380-386.

3. Cherry CL, Affandi JS, Imran D, Yunihastuti E, Smyth K, Vanar S, et al. Age and height predict neuropathy risk in patients with HIV prescribed stavudine. Neurology. 2009;73:315-320.

4. Ellis RJ, Marquie-Beck J, Delaney P, Alexander T, Clifford DB, McArthur JC, et al. Human immunodeficiency virus protease inhibitors and risk for peripheral neuropathy. Ann Neurol. 2008;64:566-572.

5. Octaviana F, Safri AY, Setiawan DD, Estiasari R, Imran D, Ranakusuma T, et al. Detectable plasma HIV RNA is associated with sensory neuropathy in HIV patients treated without stavudine. JAIDS. 2018;79(4):e108-e110.

6. Themistocleous AC, Ramirez JD, Serra J, Bennett DLH. The clinical approach to small fibre neuropathy and painful channelopathy. Pract Neurol. 2014;14:368-379.

7. Kokotis P, Schmelz M, Skopelitis EE, Kordossis T, Karandreas N. Differential sensitivity of thick and thin fibers to HIV and therapy-induced neuropathy. Auton Neurosci. 2007;136:90-95.

8. Kokotis P, Schmelz M, Papadimas GK, Skopelitis EE, Aroni K, Kordossis T, et al. Polyneuropathy induced by HIV disease and antiretroviral therapy. Clin Neurophysiol. 2013;124:176-182.

9. Lestari LK, Widyadharma PE, Merati KTP. Reliablity and validity test of neuropathic pain diagnostic questionnaire (DN4) modification to LEEDS assessment neuropathic symptoms and sign (LANSS) in HIV/AIDS patient. Neurona. 2013;30:1-6.

10. Teoh LH, Chow A, Wilder-Smith E. Skin wrinkling for diagnosing small fibre neuropathy: Comparison with epidermal nerve density and sympathetic skin response. J Neurol, Neurosurg, \& Psych. 2008;79:835-837.

11. Hitchcock SA, Meyer HP, Gwyther E. Neuropathic pain in AIDS patients prior to antiretroviral therapy. S Afr Med J. 2008;98:4:889-892.

12. Nair SN, Mary TR, Prarthana S, Harrison P. Prevalence of pain in patients with HIV/AIDS: A crosssectional survey in a South Indian State. Indian J Palliat Care. 2009;15:67-70.

13. van Hecke $\mathrm{O}$, Austin SK, Khan RA, Smith BH, Torrance N. Neuropathic pain in the general population: A systematic review of epidemiological studies: Pain. 2014;155:654-662. 
14. Mountford J, Octaviana F, Estiasari R, Setiawan DD, Ariyanto I, Lee S, et al. Ex vivo expression of chemokine receptors on cells surrounding cutaneous nerves in patients with HIV-associated sensory neuropathy: AIDS. 2018;32:431-441.

15. For the EARNEST Trial Team, Arenas-Pinto A, Thompson J, Musoro G, Musana H, Lugemwa A, et al. Peripheral neuropathy in HIV patients in sub-Saharan Africa failing first-line therapy and the response to second-line ART in the EARNEST trial. J NeuroVirol. 2016;22:104-113.

16. Chopra K, Tiwari V. Alcoholic neuropathy: possible mechanisms and future treatment possibilities: Clinical management of alcoholic neuropathy. Br J Clin Pharmacol. 2012;73:348-362.

17. Mawuntu AHP, Mahama CN, Khosama H, Estiasari R, Imran D. Early detection of peripheral neuropathy using stimulated skin wrinkling test in human immunodeficiency virus infected patients: A cross-sectional study. Medicine. 2018;97:e11526.

18. Stino AM, Smith AG. Peripheral neuropathy in prediabetes and the metabolic syndrome. J Diabetes Invest. 2017;8:646-655 


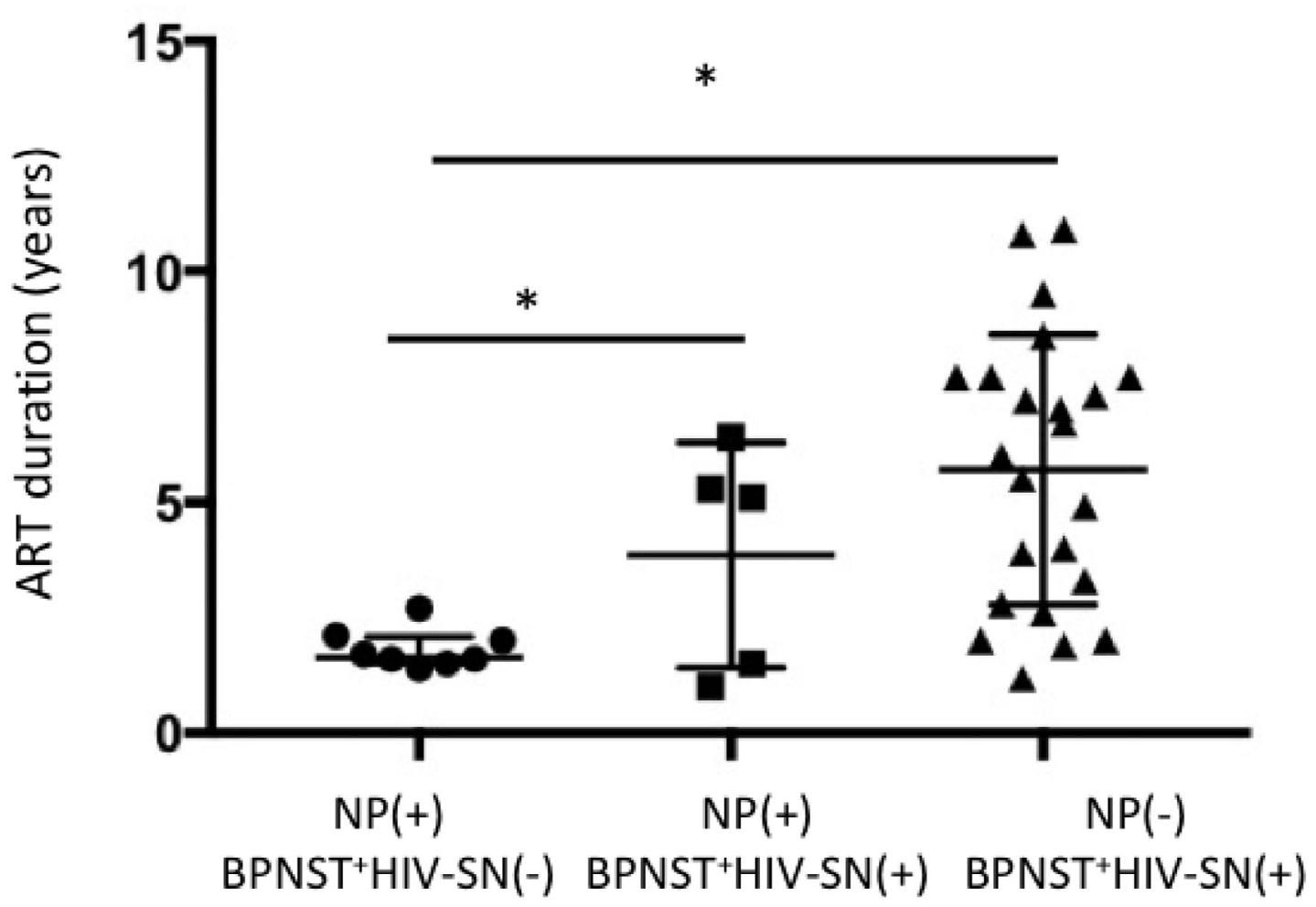


Table 1. Univariate analyses linked pain with PI use and a longer duration of ART

\begin{tabular}{|c|c|c|c|}
\hline & \multicolumn{2}{|c|}{ Neuropathic Pain } & \multirow[t]{2}{*}{$p$} \\
\hline & No $(n=184)$ & Yes $(n=13)$ & \\
\hline Female gender ${ }^{a}$ & $28.8 \%$ & $30.8 \%$ & 1.00 \\
\hline Malay ethnicity & $95.1 \%$ & $92.3 \%$ & 0.74 \\
\hline Age $\left(\right.$ years) ${ }^{b}$ & $35.8 \pm 6$ & $34 \pm 3.7$ & 0.09 \\
\hline Height $(\mathrm{cm})^{c}$ & $167(147-180)$ & 165 (142-177) & 0.58 \\
\hline Weight $(\mathrm{Kg})^{\mathrm{b}}$ & $60.4 \pm 10.5$ & $67.4 \pm 16.2$ & 0.06 \\
\hline Weight $\geq 65 \mathrm{Kg}^{\mathrm{a}}$ & $30 \%$ & $54 \%$ & 0.07 \\
\hline IVDU transmission & $36 \%$ & $38.5 \%$ & 0.6 \\
\hline Hepatitis C antibody ${ }^{a}$ & $35.3 \%$ & $30.8 \%$ & 0.50 \\
\hline Isoniazid & $45.7 \%$ & $38.5 \%$ & 0.42 \\
\hline Declared alcohol use & $22.3 \%$ & $23 \%$ & 0.59 \\
\hline >1000 copies HIV RNA/mL & $4.3 \%$ & $15.4 \%$ & 0.13 \\
\hline Nadir CD4 ${ }^{+}$T-cells/ul' & $118.5(1-599)$ & $67(10-342)$ & 0.54 \\
\hline Nadir $<200$ CD4 ${ }^{+}$T-cells/ul ${ }^{\mathrm{a}}$ & $68 \%$ & $53.8 \%$ & 0.23 \\
\hline Current CD4 ${ }^{+} \mathrm{T}$-cells/ul ${ }^{\mathrm{c}}$ & $444(21-1166)$ & $411(103-658)$ & 0.09 \\
\hline Current $<200 \mathrm{CD}^{+}{ }^{+}$-cells $/ \mathrm{ul}^{\mathrm{a}}$ & $7.6 \%$ & $23 \%$ & 0.09 \\
\hline BPNST $^{+}$HIV-SN & $12.5 \%$ & $38.5 \%$ & 0.02 \\
\hline Abnormal SSW test & $25.5 \%$ & $53.8 \%$ & 0.05 \\
\hline Abnormal NCS & $6.5 \%$ & $0 \%$ & 0.26 \\
\hline \multicolumn{4}{|c|}{ Current ART regimen } \\
\hline $2 \mathrm{NRTI}+1 \mathrm{NNRTI^{ \textrm {a } }}$ & $91 \%$ & $70 \%$ & 0.036 \\
\hline $2 \mathrm{NRTI}+\mathrm{PI}$ & $9.2 \%$ & $30 \%$ & \\
\hline Zidovudine $^{a}$ & $86.4 \%$ & $76.9 \%$ & 0.28 \\
\hline Lopinavir/Ritonavir & $10.8 \%$ & $38.4 \%$ & 0.014 \\
\hline Nevirapine & $71.2 \%$ & $69.2 \%$ & 0.55 \\
\hline Efavirenz & $44 \%$ & $69.2 \%$ & 0.07 \\
\hline Tenofovir & $32.6 \%$ & $53.8 \%$ & 0.11 \\
\hline Emtricitabine & $8.2 \%$ & $15.4 \%$ & 0.31 \\
\hline ART duration (years) ${ }^{c}$ & $4.4(1.1-12.7)$ & $1.7(1-6.4)$ & 0.002 \\
\hline ART duration $<2$ years ${ }^{a}$ & $15.2 \%$ & $53.8 \%$ & 0.002 \\
\hline
\end{tabular}

${ }^{a}$ categorical data assessed with Fischer's exact tests

${ }^{\mathrm{b}}$ mean \pm standard deviation, assessed with Student's $\mathrm{t}$ tests (parametric data)

${ }^{c}$ median (range), assessed with Mann-Whitney tests (non-parametric data)

$A R T=$ antiretroviral therapy; BPNST ${ }^{+}$-HIV-SN = HIV sensory neuropathy assessed by BPNST; IVDU = intravenous drug user; NCS =nerve conduction study; NNRTI = non nucleoside reverse transcriptase inhibitor; $\mathrm{NRTI}=$ nucleoside reverse transcriptase inhibitor; $\mathrm{PI}=$ protease inhibitor; $\mathrm{SSW}=$ stimulated skin wrinkling; 
Figure 1. The duration of ART in patients with only NP, NP and BPNST ${ }^{+} H I V-S N$ and only BPNST $^{+}$HIV-SN. Horizontal lines represent median and interquartile range. ART = antiretroviral therapy; NP = neuropathic pain; BPNST ${ }^{+}$HIV-SN = HIV sensory neuropathy assessed by BPNST. $* p<0.05$ (Mann Whitney). 